\title{
Optimization of Dangerous Heavy Gas Leakage and Diffusion Model Algorithm
}

\author{
X.M. Zheng, F.N. Yang \\ School of Computer and Information Technology \\ Beijing Jiaotong University \\ Beijing, China
}

\begin{abstract}
On the basis of research status from both at home and abroad and in view of the leakage diffusion of heavy gas stored in pressure vessels, this paper selects the heavy gas diffusion's Heavy Gas Dispersion Model in Laminar and Turbulent Atmosphere Laminar and Turbulent Atmosphere environment of Heavy Gas Dispersion Model model and multipuff Gaussian model to calculate the total amount of dangerous gas leaking from the pressure vessel into the environment based on the leakage calculation model and calculate the density of gas cloud real-timely with comprehensive consideration of time, wind speed, atmospheric stability, geographical environment and other environmental parameters. This paper aims at optimizing model algorithms to improve the model calculation speed by introducing OpenMP shared memory parallel programming under multiple CPU cores, thus calculate real-timely the density of heavy gas clouds.
\end{abstract}

Keywords-heavy gas; leakage model; LTA-HGDM model; multi-puff gaussian model; open MP parallel programming

\section{INTRODUCTION}

With the sustainable development of China's economic, the proportion taken up by chemical industry in the country's economic foundation increase constantly, meanwhile more kinds and number of dangerous chemicals gas come into being. There is great meaning to master the regularity of heavy gas's leakage and diffusion and timely predict gas diffusion to take measures to reduce disaster losses.

So far, domestic and foreign experts and scholars have developed a lot of heavy gas diffusion models such as Three-dimensional Fluid Dynamics Model, BM Model, Similar Model, Box Model and The LTA- HGDM Model as the extension model of the similar model and box model, etc. Although the calculation precision of Three-dimensional fluid mechanics model is good, the amount of calculation is large and the calculation is too complicated; BM model is consist of calculation diagrams presented according to the experimental data of heavy gas's leakage, so it is an empirical model with fine calculation accuracy and poor extensionality; Similar model and box model can only predict the general characteristics of diffusion gas clouds, regardless of the gas cloud's detailed characteristic on space; LTA HGDM (Heavy Gas Dispersion Model in Laminar and Turbulent Atmosphere Laminar and Turbulent Atmosphere environment of Heavy Gas Dispersion Model[1]) model with the basis of the box model can give out a complete description of the process of heavy gas's leakage and diffusion with the combination of the virtual point sources. Comparing with fluid mechanics calculation model and the three-dimensional finite element model, it has characteristics of small amount of calculation and high calculation precision. Therefore, the author select LTA- HGDM which is the improved from model of the Box Model to simulate the diffusion process of heavy gas clouds. As the air goes in continuously, the density of heavy gas clouds will continue to decrease and diffusion caused by the collapse of heavy gas will gradually gives way to that by environment turbulence [2], as a result, multi-puff Gaussian model is always adopted to describe the diffusion process of heavy gas clouds in late period.

In order to improve the model's calculation precision and real-timely calculate the density of heavy gas clouds diffusion in accordance with the time change at the same time, in the process of converting model calculation formula into the algorithm, the parallel calculation is introduced to improve the speed of model calculation. In multi-core era, multithread and multi-process can be adopted to realize parallel mode under stand-alone microcomputer [3].Adopting multithread means in stand-alone multi-core environment is possible because memory is shared. No access to shared data access, only the problem of data competition and lock exist. However, under multiple processes model, each process has its own storage space. In order to undertake data sharing between processes, it is necessary to achieve data relocation through communication. With comprehensive consideration of the selected calculation model of leakage diffusion, mainly is the sharing of data, and multiple processes models will increase the overhead of message passing, so the multithread means are used to undertake algorithm optimization. The Open MP programming in multithreaded take full advantage of the characteristics of the shared storage architecture, avoid the overhead of messaging and provides the parallel mechanism of fine-grained and coarse-grained. Also no big changes of codes are needed when converting serial program to parallel program except for some guidance statements, so the author choose the Open MP programming model in stand-alone environment.

\section{LEAKAGE DIFFUSION MODEL OF HEAVY GAS CLOUDS}

Diffusion analysis on dangerous chemicals accident 
involves many complicated problems, such as weather the leakage or diffusion is moment leakage or continuous leakage, the density of gas leakage, leakage direction, the phase transformation involved, the settlement of the droplets, weather conditions and nature of the ground. In order to simplify the analysis, we make the following hypothesis [2]'1, the gas clouds spread over the plain ground with no obstacles;2, neither chemical reaction and phase transformation in the gas clouds nor droplet subsidence phenomenon;3, Wind direction is the horizontal direction, and the wind's speed and direction does not change with time, place and height; 4 , no heat exchange between gas clouds and the environment.

\section{A. Leakage Calculation Model}

The speed of pressure gas leakage is associated with its flow state, and its characteristics can be described by critical flow and the subcritical flow. When it is in accordance with $\left.\frac{P_{0}}{P} \leq \frac{2}{K+1}\right)$, the gas flow belongs to velocity flow. When it meets the formula $\frac{P_{\Delta}}{P}>\left(\frac{2}{\kappa+1}\right)^{\frac{\kappa}{k-1}}$, the gas flow belongs to a subsonic gas flow. Among which, $P_{0}=$ atmospheric pressure $(\mathrm{Pa}), \mathrm{P}=$ container pressure $(\mathrm{Pa})$ and $\mathrm{k}=$ gas isentropic exponent which is the ratio between isobaric heat capacity and the constant volume heat capacity. When the gas leakage rate is approximately equal to the speed of sound, its quality leakage rate can be counted with the equation

$$
\left.Q=C_{g} A \rho\right) \sqrt{\frac{M \times\left(\frac{2}{K+1}\right)^{\frac{w+1}{N T}}}{R T}}
$$

When gas moves in subsonic flow, the leakage rate can be accounted by

$$
Q=Y C_{g} A \rho \sqrt{\frac{M \kappa\left(\frac{2}{K+1}\right)^{\frac{\kappa+1}{-1-1}}}{R T}}
$$

$Y=\sqrt{\frac{1}{\kappa-1}\left(\frac{\kappa+1}{2}\right)^{\frac{\kappa+1}{x-1}}\left(\frac{P}{P_{0}}\right)^{\frac{2}{\kappa}}\left[1-\left(\frac{P_{0}}{P}\right)^{\left(\frac{\kappa+1}{\kappa}\right)}\right]}$ of expansion factor

\section{B. Multi-Puff Gaussian Model}

When heavy gas clouds caused by leakage of dangerous gases are in late period of the diffusion in the atmosphere, and the gas clouds are continuously diluted by air with density close to air, Gaussian model can be used to simulate the gas diffusion process. According to different ways of leakage, the Gaussian model can be divided into Gaussian model and Gaussian plume model. Classic Multi-puff Gaussian model's expression[4] is:

$$
C(x, y, 0)=\frac{2 Q}{(2 \pi)^{1.5} \sigma_{x} \sigma_{y} \sigma_{z}} \exp \left[-\frac{\left(x-x_{0}\right)^{2}}{2 \sigma_{x}^{2}}\right] \exp \left[-\frac{\left(y-y_{0}\right)^{2}}{2 \sigma_{y}^{2}}\right] \exp \left[-\frac{\left(z_{0}\right)^{2}}{2 \sigma_{z}^{2}}\right]
$$

In order to improve the calculation accuracy, the gas diffusion model put forward by He Ning, etc by introducing a time factor is adopted to establish the time function to the dynamic transformation [5] as basis points. Assumes that the total time of gas leakage into the heavy gas diffusion is $\mathrm{T}$, a total number of air mass is $\mathrm{n}$ in time. At time $\mathrm{t}$, formula [6] to compute the superposition of concentration at $\operatorname{point}(\mathrm{x}, \mathrm{y}, 0)$ is

$$
C(x, y, 0)=\frac{2 Q}{(2 \pi)^{3.5} \sigma_{\alpha} \sigma_{y} \sigma_{2}} \cdot \exp \left[\frac{y^{2}}{2 \sigma_{y}^{2}}\right] \cdot \exp \left[\frac{x^{2}-u^{2}}{2 \sigma_{y}^{2}}\right] \cdot \frac{1-\exp \left[-\frac{t u x}{\sigma_{2}^{2}}\right]}{1-\exp \left[-\frac{x u}{\sigma_{2}^{2}}\right]}
$$

In which, $\mathrm{C}(\mathrm{x}, \mathrm{y}, 0)=$ the concentration at the coordinates $(\mathrm{x}, \mathrm{y})$ to the wind on the ground; $\mathrm{u}=$ wind speed, and the unit is $\mathrm{m} / \mathrm{s} ; \mathrm{t}=$ time, and the unit is $\mathrm{s} ; \mathrm{q}=$ the emissions during the accident $(\mathrm{kg} / \mathrm{s}) ;{ }_{x}, \sigma_{y}$ and $\sigma_{z}=$ the diffusion parameters (often take $\sigma_{x}=\sigma_{y}$ ) of direction $\mathrm{x}, \mathrm{y}, \mathrm{z}$, respectively.

Calculate according to Pasquill Gifford diffusion curve and Briggs expression to calculate ${ }^{\sigma_{x}},{ }^{\sigma_{y}}$ and ${ }^{\sigma_{z}}$.See table 1 for specific formula [6]:

TABLE I. SPECIFIC FORMULA.

\begin{tabular}{|l|c|c|}
\hline $\begin{array}{l}\text { atmospheric } \\
\text { stability }\end{array}$ & $\sigma_{\mathrm{x}}=\sigma_{\mathrm{y}}$ & $\sigma_{\mathrm{z}}$ \\
\hline A & $\sigma_{\mathrm{y}}=0.32 \cdot \mathrm{x} \cdot \sqrt{1+0.0004 \mathrm{x}}$ & $\sigma_{\mathrm{z}}=0.24 \cdot \mathrm{x} \cdot \sqrt{1+0.001 \mathrm{x}}$ \\
\hline B & $\sigma_{\mathrm{y}}=0.32 \cdot \mathrm{x} \cdot \sqrt{1+0.0004 \mathrm{x}}$ & $\sigma_{\mathrm{z}}=0.24 \cdot \mathrm{x} \cdot \sqrt{1+0.001 \mathrm{x}}$ \\
\hline C & $\sigma_{\mathrm{y}}=0.22 \cdot \mathrm{x} \cdot \sqrt{1+0.0004 \mathrm{x}}$ & $\sigma_{\mathrm{z}}=0.2 \mathrm{x}$ \\
\hline $\mathrm{D}$ & $\sigma_{\mathrm{y}}=0.16 \cdot \mathrm{x} \cdot \sqrt{1+0.0004 \mathrm{x}}$ & $\sigma_{\mathrm{z}}=0.14 \cdot \mathrm{x} \cdot \sqrt{1+0.003 \mathrm{x}}$ \\
\hline E & $\sigma_{\mathrm{y}}=0.11 \cdot \mathrm{x} \cdot \sqrt{1+0.0004 \mathrm{x}}$ & $\sigma_{\mathrm{z}}=0.08 \cdot \mathrm{x} \cdot \sqrt{1+0.0015 \mathrm{x}}$ \\
\hline F & $\sigma_{\mathrm{y}}=0.11 \cdot \mathrm{x} \cdot \sqrt{1+0.0004 \mathrm{x}}$ & $\sigma_{\mathrm{z}}=0.08 \cdot \mathrm{x} \cdot \sqrt{1+0.0015 \mathrm{x}}$ \\
\hline
\end{tabular}

\section{LTA-HGDM Model}

Assuming that the cloud radius is $\mathrm{R}$ and the height is $\mathrm{h}$, it is believed that cloud's static pressure is equal to the power of the air drag, and thus the radial size changing rate of the cloud clusters [8] is:

$$
\frac{d R}{d t}=a_{1} b^{0.5} / R
$$

Among which: $b=g V \Delta / \pi$. In cases that isothermal flow or diffusion gas has the same molar heat with air and nonisothermal flow cases in which ground heating can be ignored, the value of $\mathrm{b}$ can be approximately equal to $b_{0}$. $b_{0}=g V_{0} \Delta_{0} / \pi$. In which, Integrate equation (5) to get:

$$
R^{2}=R_{0}^{2}-2 a_{1} b^{1.5} t
$$

Cloud's volume is as follows: and:

$$
\begin{aligned}
& \frac{V}{V_{0}}=\left(1+c_{1}\left(\frac{H_{0}}{D_{0}}\right)^{1 / 3}(t / \tau)^{2}\right)^{C_{2}} \\
& \tau=\left(\frac{V V_{0} / 3}{g \Delta \Delta_{0}}\right)^{1 / 2}
\end{aligned}
$$

In the process of heavy gas clouds spread, as the air continuously come in, the edge of the cloud is continually diluted by air and presents the Gaussian distribution, and air entrainment effect is not immediately influenced inside the cloud. All the assumptions are within the range of radius, and the gas cloud concentration distribution is uniformity. At this time the concentration can be expressed as:

$$
C=C_{0} V_{0} / V e^{-z^{2} / 2 \sigma_{z}^{2}} \cdot e^{-\left(r-R_{c}\right)^{2} / 2 \sigma_{r}^{2}} \quad r>R_{c}
$$




$$
C=C_{0} V_{0} / V e^{-z^{2} / 2 \sigma_{z}^{2}} \quad r<R_{c}
$$

$R_{c}$ can be resolved by solution 12 :

$$
R_{c}=R_{c}^{2}+2 \pi^{0.5} R_{c \sigma_{r}}+2 \sigma_{r}^{2}
$$

Due to the fact that the diffusion of heavy gas clouds is under the environment of atmospheric turbulence, so the heavy gas diffusion coefficient comes from two aspects of turbulent diffusion caused by subsidence and environmental turbulence diffusion [7]:

$$
\begin{aligned}
& \sigma_{r}^{2}=\sigma_{r g}^{2}+\sigma_{r a}^{2} \\
& \sigma_{z}^{2}=\sigma_{z g}^{2}+\sigma_{z a}^{2}
\end{aligned}
$$

It is generally believed that cloud height is the height of the cylinder in box model ${ }^{[1]}$, namely: $\sigma_{z}=(2 / \pi)^{1 / 2}$ and $\sigma_{r g}=a_{2}\left(R-R_{0}\right)$. On the calculation of $\sigma_{r a}$, use the following formula:

$$
\sigma_{r a}=U \cdot t / \tau q R i i_{1}^{1 / 2} \cdot \Phi /(1+\Phi)
$$

And

$$
\Phi=\pi \frac{R i_{c}}{R i \pi(R / L)^{2}}\left(R_{0} / L+\frac{a_{i}}{\sqrt{\pi}}\left(\frac{t}{\tau}\right)\right)
$$

In the process of heavy gas clouds spread, as the air continuously come in, the edge of the cloud is continually diluted by air and presents the Gaussian distribution, and air entrainment effect is not immediately influenced inside the cloud.. Judgment of weather heavy gas clouds shift to the heavy gas clouds of points can use the rule of $\mathrm{Ri}$ : when the $\mathrm{Ri}$ is less than the critical Richardson number, heavy gas clouds convert into non heavy gas clouds [9], and the gas cloud diffusion model transformation convert into Gaussian model. Define the Richardson number as follows:

$$
R i=\left[g\left(\rho_{0}-\rho_{a}\right) / \rho_{a}\right] V_{0} / V^{2 / 3} V_{*}
$$

Among which: $V_{*}=$ friction velocity, and the unit is $\mathrm{m} / \mathrm{s}$. It is related to wind speed, ground roughness and atmospheric stability, which is approximately equal to $1 / 15$ of $10 \mathrm{~m}$ high wind speed. Preliminary determine according to wind speed: when $\mathrm{U}>4, V_{f}=5 / 15$; when $3<\mathrm{U} \quad 4, V_{f}=$ 4/15; when $\mathrm{U} \leq 3, V_{f}=3 / 15$.

The significance of symbols in each formula above is as following: $\mathrm{R}_{\mathrm{c}}$ is the cloud core radius; $\mathrm{H}$ is cloud height; $\mathrm{V}$ is the cloud volume, $\rho_{0}$ is initial density of the cloud, $V_{0}$ is the initial volume of the cloud, $\mathrm{H}_{0}$ is cloud's initial height, $\mathrm{T}$ is cloud's diffusion time, $\mathrm{L}$ is the feature of cloud sizes, $\mathrm{C}$ is cloud's internal Moore coefficient; $\sigma_{\mathrm{rg}}$ is radial diffusion coefficient caused by gravity settling, $\sigma_{\mathrm{zg}}$ is the vertical diffusion coefficient caused by gravity sedimentation, and the unit is $m ; a_{1}$ is the cloud's gravity drop coefficient; $\mathrm{Ri}_{c}$ is a critical Richardsion number, in this paper, the values of 8 ; $\mathrm{Ri}$ is Richardsion number; $\mathrm{R}$ is cloud radius; $\mathrm{g}$ is the gravitational acceleration; $\rho_{a}$ is the air density; $R_{0}$ is initial radius of the cloud; $D_{0}$ is initial diameter of the cloud; $\Delta_{0}$ is the initial density difference between clouds and the surrounding air; $\tau$ is features of cloud diffusion time; $r$ and $\mathrm{z}$ are cylindrical coordinates of predicted points, $\sigma_{\mathrm{ra}}$ is the vertical diffusion coefficient caused by gravity sedimentation; $\sigma_{\mathrm{za}}$ is the cloud's gravity drop coefficient; $\mathrm{Ri}_{1}$ is a critical Richardsion number; $\mathrm{U}$ is the environment wind speed.

\section{ALGORITHM OPTIMIZATION AND IMPLEMENTATION}

Open MP is programming model based on the thread. Its execution model adopts the Fork - Join form . The Fork Join execution mode at the time of start, there is only one is called "the main thread" running threads. In the running process of the main thread, when faced with the need for parallel computing, it derived the thread to perform the tasks in parallel. At the time of parallel execution, the main thread and spawned thread work together. At the end of the parallel code executed, spawned thread exit or hang up, not in work, and the control process come back to the separate main thread [10].

Pseudo codes which the Algorithm designs are as follows:

(1) Input environment atmosphere pressure, pressure container, vessel rupture area, dangerous gas temperature and take use of the leakage calculation model to calculate the gas leakage quantity $\mathrm{Q}$ within a certain time range.

(2) According to the time change, real-timely calculate the concentration of dynamic computing clouds. For a period of time, the scope of the concentration of cloud is accumulative value of each point cloud concentration. Join \# romp parallel and \# pragma omp for reduction (+: condensity) into the main thread to loop parallelization compiler guidance statements. Condensity is accumulated value of concentration, because the first time Idensity depends on the $(i-1)$ cycle as a result, use the reduction to reduce circular dependencies.

(3) Call \# pragma omp parallel sections num threads statement (3) compiled instruction, and create three parallel threads. In the parallel area, call \# pragma omp section to compile guidance statements, in the process of heavy gas clouds are calculated separately, and the diffusion radius $\mathrm{R}$ and friction velocity and volume $\mathrm{V}$.

(4) Call \# pragma omp barrier compilation instructions to calculate the value of Ri. Judge the relative size between $\mathrm{Ri}$ value and the number of critical Richardson. If $\mathrm{Ri}<$ Richardson number, the program execute step (7) and (8). Otherwise, it executes the first step (5) and (6).

(5) Call again \# pragma omp parallel sections statements, and open three threads, to calculate intermediate, variable number, respectively.

(6) Again call \# pragma omp barrier compile statements which synchronize the threads. Call the \# pragma omp parrllel the statement (val) instruction, calculate the heavy gas cloud density according to the $\mathrm{r}$ value and the relative size. 
(7)Split the formula (5) into five parts:

$$
\begin{aligned}
& \frac{2 Q}{(2 \pi)^{1.5} \sigma_{x} \sigma_{y} \sigma_{z}} \quad, x p\left[\frac{y^{2}}{2 \sigma_{y}^{2}}\right], x p\left[\frac{x^{2}-u^{2}}{2 \sigma_{y}^{2}}\right], 1-\exp \left[-\frac{t u x}{\sigma_{x}^{2}}\right], \\
& 1-\exp \left[-\frac{x u}{\sigma_{x}^{2}}\right]
\end{aligned}
$$

Call the \# pragma omp parallel sections, put each part into the different threads to calculate the value. Put the calculated value of each part in the array result.

(8) Call \# pragma omp parrllel for reduction (+:condensity) compilation guidance statements, and dynamically calculate clouds Gaussian density.

(9) In the end, save the instantaneous density of the cloud in the queue as input data to front desk $3 \mathrm{~d}$ simulations.

According to the designed algorithm, we run test respectively on two dual-core CPU and 12 PCs with same memory and the same type of CPU type. Set initial values for other leakage phases, pressure in the vessel is $1.17 \times 10^{7}$ $\mathrm{Pa}$, environmental pressure is $1.01 \times 10^{7} \mathrm{~Pa}$, crack area is 7.85 $\times 10^{-7} \mathrm{~m}^{2}$, crack shape is circular. The temperature of the gas is 30 degrees Celsius. Adiabatic index of the gas is 1.31 . Clouds initial density is $3.133 \mathrm{~kg} / \mathrm{m}^{3}$, air density is 1205 $\mathrm{kg} / \mathrm{m}^{3}$, atmospheric stability is in "A" type, the cloud initial height is 3 , the environment wind speed is $3 \mathrm{~m} / \mathrm{s}$, ground roughness type is grasslands, open and plain ground.

First of all, compare between calculated results without using parallel computing with ordinary calculation, the time difference is shown in figure 1 :

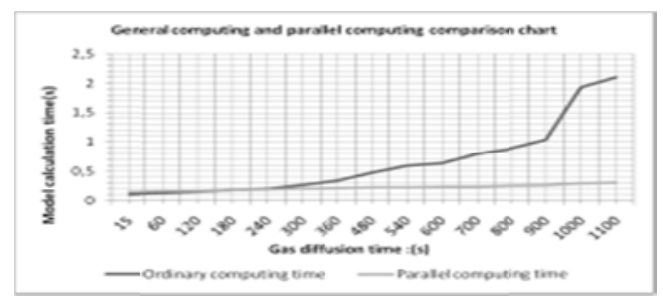

FIGURE I. COMPARING FIGURE BETWEEN TWO CALCULATION DIAGRAMS

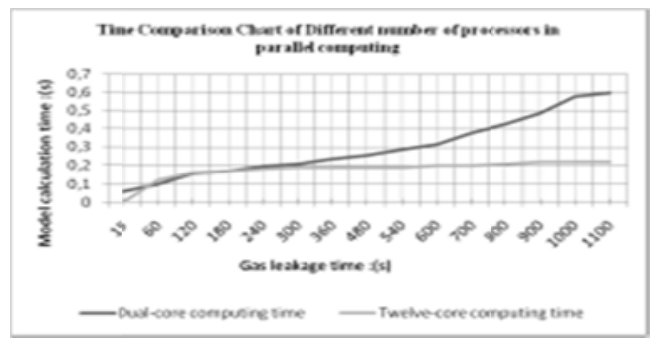

FIGURE II. COMPARING FIGURE OF PARALLEL COMPUTING UNDER DUAL-CORE PROCESSOR AND 12 CORE PROCESSOR.

Analysis from the graph, we can know that when the diffusion time is short, parallel computing time is bigger than ordinary calculation. This is because in the process of the parallel computing, the tasks' asynchronous execution requires a certain time. With the increase of gas cloud diffusion time, the time parallel computing method requires for computing the result has no obvious change, while the time calculation methods without using parallel computing needs increase gradually with diffusion time. Under dual core and twelve nuclear environment, time comparison between model calculation is shown in figure 2 .

As shown in figure 2, at the time of initial diffusion, 12 nuclear PC's computing speed is obviously faster than dualcore PC. This is because the amount of calculation is small and data competition is small. The more the processor, the faster the calculation speed; With the increase of diffusion time, the number of threads 12 core processor PC system requires to deal with is 6 times the dual-core processor $\mathrm{PC}$, so it needs to for assign tasks for number of threads, which takes some time; When diffusion time continues to increase and the computation grows, 12 core processor's scheduling becomes stabilize, and computing time tends to be stable; The dual-core processor, because of the increased amount of task, takes longer time than 12 core processor. It can be seen from the diagram that the longer the gas diffusion time and the larger the number of processors, the better the algorithm results after optimized by parallel computing model.

\section{CONCLUSION}

Calculate the leaking amount of dangerous heavy gas from storage tank into the atmosphere through adopting the leaking model. According to the material leakage amount, combined with environmental parameters such as the environmental wind speed, atmospheric stability, surface rough type parameters, according to the diffusion time, predict the gas cloud density in downwind distance. Because the heavy air inhaled air continuously in the diffusion process and convert into the heavy gas clouds, within a certain time, gas cloud is heavy gas clouds. Over a particular time value, gas cloud will diffuse in gauss methods. By introducing a multi-threaded parallel computing, the time of the whole model calculation is shorter. The next step of work, the author will further research the combination between the heavy gas clouds diffusion calculation and $3 \mathrm{~d}$ virtual reality environment, through the virtual reality environment, show the whole process of diffusion that dangerous heavy gas leak from the storage tank into a specific environment.

\section{ACKNOWLEDGMENT}

"The system platform of railway transportation safety management of domestic LINUX server operating system based on" The Ministry of science and technology "nuclear high based" major project number: 2010ZX01036-001-001.

\section{REFERENCES}

[1] Yan.Shanyu, Panke. 2009. Development and application of Inflammable, explosive and toxic clouds spread software based on JAVA technology.China Safety Science Journal: 152-158.

[2] Xu, Zhisheng \& Jiang, Xuepeng \& Wu, Chao. 2012. Beijing. Safety system engineering. Machinery industry press: 216-251.

[3] Intel Asia Pacific r\&d co., LTD. 2010.Beijing. Beijing Parallel science and technology co., LTD. Release potential of multicore, Intel Parallel Studio Parallel development guide. Tsinghua university press.

[4] Liu, Tiemin, \& Zhang, Xing kai \& Liu,Gongzhi. 2005. Beijing. Safety evaluation method and application guide . Chemical industry press. 
[5] Yuan, Danan \& Li, Shigang \& Huang, Yicong \&Wu Mingxia.2013. Petrochemical gas leakage diffusion model research progress. Journal of chemical industry and engineering technology: 21-26.

[6] He, Ning. 2009. Toxic gas diffusion model in the application of the accident rescue. Journal of natural disasters: 197-200.

[7] He, Ning \& WU,Zongzhi \&Zheng,Wei.2010. Simulation of an Improved Gaussian Model for Hazardous Gas Diffusion.JOURNAL OF BASIC SCIENCE AND ENGINEERING 18(4):571-579.

[8] Jang, Juncheng \& Pan, Xuhai.2001. The new model for describing the heavy gas leakage diffusion process. Journal of nanjing university of technology : 41-46.

[9] Yu, Lijian. 2013. Study on a moment of heavy gas dispersion model. Journal of safety science and technology of China 9(1):12-16.

[10] Bibli Multi-core series materials editorial. Multi-core programming.2007. Beijing. Tsinghua university press. 